Revista Interdisciplinaria de Humanidades, Educación, Ciencia y Tecnología Año VII. Vol. VII. №13. Julio - Diciembre. 2021

Hecho el depósito de ley: pp201602FA4721

ISSN-L: 2542-3029; ISSN: 2610-802X

Universidad Nacional Experimental Francisco de Miranda (UNEFM). Santa Ana de Coro. Venezuela

Marcos Damián Vivar-Vicuña; Juan Carlos Erazo-Álvarez

DOI $10.35381 / \mathrm{cm} . \mathrm{v} 7 \mathrm{i} 13.480$

\title{
Enseñanza en la Educación Básica Superior: Una experiencia en Classroom
}

Teaching in the Basic Superior: An experience in Classroom

\author{
Marcos Damián Vivar-Vicuña \\ mdvivarv75@est.ucacue.edu.ec \\ Universidad Católica de Cuenca, Azogues \\ Ecuador \\ https://orcid.org/0000-0001-8283-1550 \\ Juan Carlos Erazo-Álvarez \\ jcerazo@ucacue.edu.ec \\ Universidad Católica de Cuenca, Cuenca \\ Ecuador \\ https://orcid.org/0000-0001-6480-2270
}

Recibido: 10 de marzo de 2021

Aprobado: 15 de junio de 2021 


\author{
CIENCIAMATRIA \\ Revista Interdisciplinaria de Humanidades, Educación, Ciencia y Tecnología \\ Año VII. Vol. VII. N¹3. Julio - Diciembre. 2021 \\ Hecho el depósito de ley: pp201602FA4721 \\ ISSN-L: 2542-3029; ISSN: 2610-802X \\ Universidad Nacional Experimental Francisco de Miranda (UNEFM). Santa Ana de Coro. Venezuela
}

Marcos Damián Vivar-Vicuña; Juan Carlos Erazo-Álvarez

\title{
RESUMEN
}

La tecnología ha obligado a la educación a familiarizarse más con los recursos tecnológicos de manera sincrónica y asincrónica, aprendiendo a convivir con la virtualidad, como lo es el uso de la plataforma Google Classroom, preguntándose si es necesario explicar el uso de la plataforma, realizando un análisis no experimental, con un enfoque cuantitativo y de cohorte transversal. El objetivo de la investigación es explicar de qué manera el uso de la plataforma puede aportar al aprendizaje de los estudiantes de Educación Básica Superior de la escuela 4 de noviembre de la ciudad de Azogues. El instrumento aplicado a los estudiantes fue la encuesta, donde de acuerdo a resultados la totalidad de la población determina que desea utilizar Classroom en el proceso de enseñanza. Por tal motivo la investigación es de carácter relevante para la educación siendo así un aporte para la ciencia y la tecnología.

Descriptores: Enseñanza asistida por ordenador; aprendizaje en línea; educación inicial. (palabras tomadas del Tesauro UNESCO).

\begin{abstract}
Technology has forced education to become more familiar with technological resources synchronously and asynchronously, learning to live with virtuality, as is the use of the Google Classroom platform, wondering if it is necessary to explain the use of the platform, conducting a non-experimental analysis, with a quantitative and crosssectional cohort approach. The objective of the research is to explain how the use of the platform can contribute to the learning of the students of Higher Basic Education of the 4 de November school in the city of Azogues. The instrument applied to the students was the survey, where according to the results the entire population determines that they want to use Classroom in the teaching process. For this reason, research is relevant for education, thus being a contribution to science and technology.
\end{abstract}

Descriptors: Computer assisted instruction; electronic learning; initial education. (words taken from the UNESCO Thesaurus). 


\section{CIENCIAMATRIA \\ Revista Interdisciplinaria de Humanidades, Educación, Ciencia y Tecnología \\ Año VII. Vol. VII. N¹3. Julio - Diciembre. 2021 \\ Hecho el depósito de ley: pp201602FA4721 \\ ISSN-L: 2542-3029; ISSN: 2610-802X \\ Universidad Nacional Experimental Francisco de Miranda (UNEFM). Santa Ana de Coro. Venezuela}

Marcos Damián Vivar-Vicuña; Juan Carlos Erazo-Álvarez

\section{INTRODUCCIÓN}

A partir del siglo XXI la tecnología ha ido apropiándose de la sociedad, ya sea de forma directa $O$ indirecta, las necesidades de las experiencias de aprendizajes virtuales giran alrededor del mundo cuestionando el valor de aprendizajes significativos entorno a la comunidad, tomando en cuenta los aportes de Cano y Olda (2012). En el campo educativo ha tenido un impacto positivo, puesto que por medio de plataformas como es Google Classroom se ha podido tener procesos de enseñanza-aprendizaje, profesores-alumnos. Esta realidad virtual a nivel global según Aznar et al. (2018) argumenta que se ha instaurado de manera sorprendente ya en la actual sociedad a nivel educativo.

Entre los grandes retos de América latina ha sido la incorporación de nuevas tecnologías para el análisis de diversos temas educativos mencionando a Peña y Otálora (2018) que plantean modernizar la educación, acelerando el avance científico y tecnológico, afianzando la capacidad para el crecimiento educativo y económico, garantizando el desarrollo de la sociedad. Considerado como un contenido que se denomina un desafío de las Tecnologías de la Información y Comunicación (TIC). Por lo consiguiente Hernández (2017) expresa que el conocimiento ha traído cambios, el resultado ha sido en masa y multiplicador, siendo que estas han ido formando parte de la vida diaria de la sociedad latinoamericana.

Las tecnologías en la educación ecuatoriana se han visto afectadas por cuanto al desconocimiento por parte de docentes y estudiantes del uso y manejo de plataformas y aplicaciones, debido que es una sociedad que no estuvo acostumbrada a la era de las tecnologías. Se puede adicionar a los autores Navarrete y Mendieta (2018) en donde afirman que la nueva era tecnológica obliga en el ámbito educativo a cambiar desde sus bases para conseguir en los estudiantes resultados positivos aportando el desarrollo de la sociedad educativa nacional.

Por último, la educación como se refiere Paladines (2016) analiza que el sistema educativo hace años atrás en el siglo XX. Ecuador estuvo inmerso en una crisis económica y educativa; y en procesos incompletos. Hoy en la actualidad la educación tecnológica se ha reinventado mediante las plataformas y aplicaciones como Google 


\section{CIENCIAMATRIA \\ Revista Interdisciplinaria de Humanidades, Educación, Ciencia y Tecnología \\ Año VII. Vol. VII. NN13. Julio - Diciembre. 2021 \\ Hecho el depósito de ley: pp201602FA4721 \\ ISSN-L: 2542-3029; ISSN: 2610-802X \\ Universidad Nacional Experimental Francisco de Miranda (UNEFM). Santa Ana de Coro. Venezuela}

Marcos Damián Vivar-Vicuña; Juan Carlos Erazo-Álvarez

Classroom, que se ha venido adaptando al medio educativo y ha formado parte de la vida estudiantil como laboral, debido que esta plataforma ayudará al desempeño de manera eficiente.

Se realizará esta investigación para fomentar en la sociedad el uso y conocimiento de un aula virtual como es Google Classroom; dando a conocer las ventajas que conlleva en el proceso de enseñanza-aprendizaje de docentes y estudiantes. Las plataformas son la innovación educativa del momento creando una gran ayuda tanto a nivel académico como laboral para así fortalecer los conocimientos y lograr una enseñanzaaprendizaje en el alumnado mejorando sus expectativas de calidad en las diferentes áreas del conocimiento.

A nivel de la sociedad aportara una comunicación más efectiva puesto que es un avance a nivel de la población ecuatoriana, para dar las herramientas a los estudiantes para que puedan descubrir varios usos que aporten a la ciencia. Se puede mencionar que entre los usos y beneficios de la plataforma Google Classroom está el que se puede subir y descargar información que el usuario lo desea, como a su vez trabajar de manera colaborativa entre varias personas, cabe manifestar que también sirve como una nube de almacenamiento masivo que es utilizada hoy por hoy a nivel global. Entre los aspectos más importantes se conoce que es una plataforma de fácil acceso por partes de docentes, estudiantes y cualquier tipo de usuario y gratuita utilizando simplemente un correo electrónico de Google pensando en mejorar la relación de aprendizaje como hace referencia la Ley Orgánica de Educación Intercultural [LOEI] (2013) considerando al inter-aprendizaje y multi-aprendizaje como herramientas para maximizar las capacidades humanas y tecnológicas a treves de la sociedad, el acceso a la información y sus tecnologías, la comunicación y el conocimiento, para lograr el desarrollo de los estudiantes como docentes de forma individual y colectiva.

La plataforma Google Classroom contribuirá beneficiando a la sociedad educativa en cuanto es de fácil acceso desde cualquier medio tecnológico (computadora, celular, Tablet, etc.) de esa manera poder cargar y descargar archivos de su necesidad. Se puede aportar la utilización de aulas virtuales para el mejor desempeño a los estudiantes y docentes en las instituciones educativas públicas como privadas como 


\section{CIENCIAMATRIA}

Revista Interdisciplinaria de Humanidades, Educación, Ciencia y Tecnología

Año VII. Vol. VII. N¹3. Julio - Diciembre. 2021

Hecho el depósito de ley: pp201602FA4721

ISSN-L: 2542-3029; ISSN: 2610-802X

Universidad Nacional Experimental Francisco de Miranda (UNEFM). Santa Ana de Coro. Venezuela

Marcos Damián Vivar-Vicuña; Juan Carlos Erazo-Álvarez

manifiesta la [LOEI] (2013) garantizar la alfabetización digital y el uso de las tecnologías de la información y comunicación, siendo de esta forma un beneficio hacia la educación de la escuela de Educación General Básica 4 de Noviembre de la ciudad de Azogues, provincia del Cañar.

\section{Referencial teorico}

Por medio de otras investigaciones de diferentes autores se refieren a que el fenómeno de la educación por medio de la tecnología ha tenido gran acogida hace muchos años atrás debido que es una herramienta muy eficiente a nivel global permitiendo conectarse por medio de la tecnología con la humanidad sin importar la cultura que con lleva cada una de estas, como menciona Aznar et al. (2018) refiriéndose que el interés de la temática es creciente y la universalización de los dispositivos tecnológicos está permitiendo el acceso a la Realidad Virtual a todo el público facilitando la conectividad global.

La globalización de la tecnología ha ido desarrollando transformaciones y adaptaciones en la educación como expresa Cano y Olda (2012) que los aspectos controversiales de la calidad académica, involucra una transformación de contenidos pedagógicos y didácticos en la presentación y adquisición de la información, en las habilidades de aprendizaje del estudiante. Asumiendo en la actualidad retos a nivel global obligando a la población a auto educarse o por medio de tutoriales y cursos para su correcto funcionamiento y poder llegar a cada uno de los estudiantes sin importar la situación geográfica.

A nivel de latinoamericano se puede referenciar a Poma (2019) que afirma que el estudiante en Perú se tiene que preparar para poder seguir compitiendo, mediante el uso herramientas digitales, como es la plataforma Google Classroom del campo virtual de la universidad Nacional de Huancavelica. Se observa que ha tenido un gran crecimiento el uso de las plataformas en el ámbito educativo a medida que en países como Argentina, Chile, Venezuela ha ido evolucionando a la educación constantemente gracias a la tecnología. 


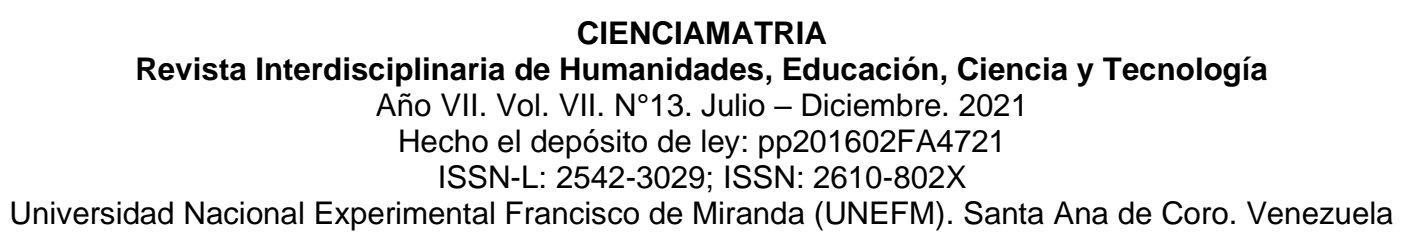

Marcos Damián Vivar-Vicuña; Juan Carlos Erazo-Álvarez

En el ámbito de la educación nacional se ha dado una mínima restricción debido que el poder de adquisición por la sociedad ecuatoriana es bajo especialmente en los sectores rurales que es de difícil acceso al internet por motivos de estos no se ha podido llegar a una educación igualitaria debido a este factor que muy difícil se va a lograr superar, siendo a su vez las únicas oportunidades que les faciliten en instituciones educativas que tengan acceso a estas plataformas y al uso de internet. De acuerdo con Navarrete y Mendieta (2018) manifiesta que las herramientas digitales potencializan el desarrollo intelectual, ayuda en la innovación del aprendizaje, para la adaptación de nuevas estrategias para la enseñanza-aprendizaje. Teniendo como resultado la maximización de las habilidades cognitivas del individuo ya sean niños, jóvenes o adultos para su desarrollo en el campo educativo y aprovechamiento de sus nuevas habilidades tecnológicas que se va ir creando en el estudiante o el individuo en las diferentes áreas.

Y sin embargo Aznar et al. (2018) constata que en varios niveles educativos que se ha dado la necesidad para su desarrollo para ello surgió la implementación universal de dispositivos inteligentes y abaratamientos de equipos virtuales. La implementación de dispositivo ha generado ventajas porque ha permitido la conexión de las personas sin importar el lugar en donde se encuentren, también el desarrollo educativo puesto que por medio de la plataforma se puede estudiar sin importar su lugar geográfico, como también se puede utilizar como dispositivo de almacenamiento y desventaja en cuanto al poder adquisitivo económico porque cada individuo se ve en la obligación a la adquisición de uno de estos dispositivos.

En esta investigación se menciona a las habilidades de enseñanza aprendizaje en donde el aprendizaje son los conocimientos adquiridos del individuo a diario, en cuanto al papel que juega el aprendizaje Monge (2014) menciona que los docentes intercambian ideas de su experiencia en sus clases y enfocan los procesos de aprendizaje de sus estudiantes, generalmente hablan del entusiasmo y empatía hacia esta materia y un gusto por hacer lo aprendido.

La educación viene dando cambios gigantescos a nivel global como lo menciona Rodríguez et al. (2013) que la educación es la nueva herramienta para actuales y 


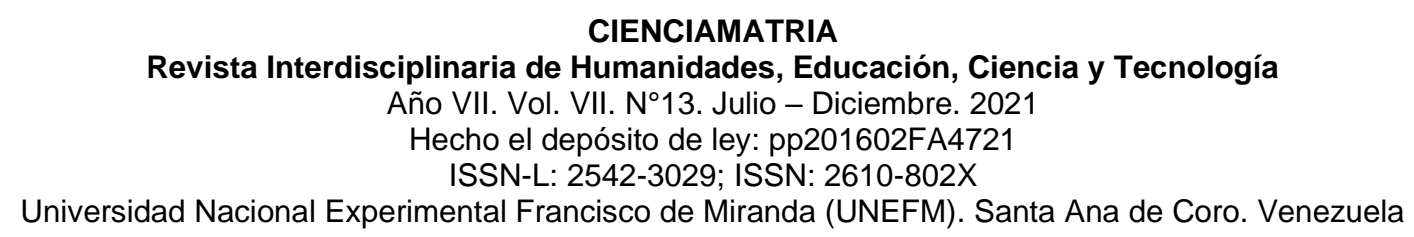

Marcos Damián Vivar-Vicuña; Juan Carlos Erazo-Álvarez

futuras generaciones, en donde hay que prepararse primeramente a nivel educativo para poder competir en otros ámbitos, como también es un arma para poder ser mejores ante otros países más desarrollados puesto que por medio de la tecnología se puede innovar de cualquier lugar del mundo.

El individuo siempre está en la capacidad de aprender y cada día se aprende algo nuevo mediante un intercambio de vivencias que se da en el día a día ya sea en el ámbito educativo como social creando procesos de enseñanza aprendizaje, partiendo siempre de mejorar la calidad de la educación desde mínimos espacios hasta avanzar a nivel internacional o de otra manera que se le podría llamar es de manera global llegando por medio de la tecnología a todo sitio geográfico.

La enseñanza asincrónica brinda la posibilidad de que la educación se habilite de una manera didáctica, con la utilización de diferentes tipos de metodologías según Tabarez (2010) menciona que el campo de la virtualita se encuentra enlazados a los espacios asincrónicos para total comunicación de los estudiantes y de los profesores en varias etapas de la educación que se le puede apreciar en la actualidad con el afán de sacarle el mejor provecho a la tecnología.

La educación a distancia desde el punto de vista de Páez-Barón et al. (2016) se restringía a unos procesos de transferencia de conocimientos de los docentes a los estudiantes, usando medios de comunicación que podían demorar ciertos días en llegar al receptor; se constituía básicamente en un modelo de comunicación con una sola dirección y escasa interacción, basado en el desarrollo de tareas determinadas de acuerdo al tiempo programado por cada docente o administrador.

Actualmente, los procesos de comunicación entre docentes y estudiantes desde la educación virtual se realizan prácticamente de manera instantánea y en tiempo real, logrando el desarrollo de un proceso formativo más dinámico y participativo en el que se ven involucrados múltiples actores: el docente, los alumnos y la comunidad educativa, entre otros, también podemos destacar que se puede trabajar varios colaboradores en un mismo documento al mismo tiempo.

La educación Básica Superior se le considera como un subnivel de la Educación General Básica comprendido en la actualidad en octavo, noveno y décimo año 


\section{CIENCIAMATRIA}

Revista Interdisciplinaria de Humanidades, Educación, Ciencia y Tecnología

Año VII. Vol. VII. N¹3. Julio - Diciembre. 2021

Hecho el depósito de ley: pp201602FA4721

ISSN-L: 2542-3029; ISSN: 2610-802X

Universidad Nacional Experimental Francisco de Miranda (UNEFM). Santa Ana de Coro. Venezuela

Marcos Damián Vivar-Vicuña; Juan Carlos Erazo-Álvarez

respectivamente entre las edades promedio de 15 y 16 años considerando a la ordinaria o presencial, como también hay diferentes modalidades de estudio como: son proyectos intensivos, semi-presencial, a distancia y proyectos intensivos designados para diferentes edades, pero con el mismo propósito que es la Básica Superior.

Se llevará a cabo la aplicación de una metodología activista conforme dice Puga y Jaramillo (2015) que ayuda a maximizar el desarrollo del conocimiento, buscando a que el estudiante permanezca en constante participación, para lograr darle fuerza al aprendizaje significativo. La metodología activa se basa principalmente en la comunicación del profesor con el alumno, el alumno con otro alumno, o el alumno con el profesor y se puede llevar a cabo de igual manera a través de un recurso digital como es la plataforma Google Classroom.

De esta manera Google Classroom es una herramienta digital creada para el beneficio exclusivamente hoy en día de la educación, a partir de esta plataforma se puede ir destacando en la misma que, va avanzando para el bienestar de los alumnos en la enseñanza aprendizaje siendo esta un aula virtual, utilizando varias metodologías e instrumento adaptándolos al medio de trabajo siendo esta una manera de incorporación en la sociedad educativa.

Por medio de un internet nos permite compartir documentos con todos los usuarios, como plantea Gómez y Valdivia (2020) señalando que es una aplicación que permite crear aulas virtuales, estableciendo objetivos y cumplimientos mejorando la comunicación entre docentes y estudiantes, además que los trabajos se guardan de manera ordenada en Google Drive, con disponibilidad para cualquier tipo de persona y sin tener que pagar ningún otro valor adicional a lo normal para su funcionamiento. A partir de esta plataforma vamos a obtener las clases, tareas y facilidad en el uso de esta herramienta facilitando el trabajo del docente, de esta manera permite gestionar las clases virtuales, y hasta poder hacer una evaluación y obtener la calificación de manera inmediata, para los alumnos es una manera eficaz de utilizar esta plataforma permitiéndoles acceder desde cualquier dispositivo a sus clases esta es la principal ventaja de la plataforma Google Classroom. 


\section{CIENCIAMATRIA \\ Revista Interdisciplinaria de Humanidades, Educación, Ciencia y Tecnología \\ Año VII. Vol. VII. N¹3. Julio - Diciembre. 2021 \\ Hecho el depósito de ley: pp201602FA4721 \\ ISSN-L: 2542-3029; ISSN: 2610-802X \\ Universidad Nacional Experimental Francisco de Miranda (UNEFM). Santa Ana de Coro. Venezuela}

Marcos Damián Vivar-Vicuña; Juan Carlos Erazo-Álvarez

De acuerdo a Cacheiro (2011) destaca que varios de los recursos digitales o herramientas tecnológicas de aprendizaje entre los cuales se puede nombrar a varios de ellos: repositorios de recursos educativos, tutoriales interactivos, grabaciones multimedia como: audios, videos, imágenes; cuestionarios online, herramientas web 2.0 como: eBooks, Podcast, etc.; y cursos en línea dictados, cada uno de ellos aportan de manera directa a la educación.

Es por ello que estos recursos digitales aportan con el uso de la plataforma Classroom porque como bien ya se menciona es una gran plataforma de mucha utilidad en la actualidad, donde se puede seguir cada una de las etapas para la construcción de una clase, muy completa en todo abarca un sin número de recursos en uno solo facilitando un trabajo activo entre los usuarios estudiantado y profesores miembros de la comunidad tecnológica educativa.

En la realidad ecuatoriana la plataforma Google Classroom ha sido la herramienta más utilizada que se ha implementado en la educación, es tal el auge de esta plataforma que se utiliza desde los niños de temprana edad, jóvenes y hasta adultos en escuelas colegios y universidades, sin importar la edad ya sea por medio de videos explicativos y de igual forma se ha adaptado para lo que es todo tipo de educación presencial, semipresencial, virtual o en línea aportando a la comunicación activa de los usuarios de la misma, generando la ampliación del conocimiento.

Por último la plataforma aporta a la escuela de Educación General Básica 4 de Noviembre, un registro de las actividad de los estudiantes que envían, a su vez queda la evidencia para respaldo ya se de los estudiantes como de los profesores, también se programa a la plataforma para evaluar la tarea subida por parte del estudiante y ser calificada de acuerdo a los parámetros dispuestos por el profesor de esta manera facilita en cuanto al tiempo utilizado es mínimo comparado a otros métodos y también aporta al medio ambiente sin la utilización del papel.

\section{METODOLOGÍA}

Este fenómeno tecnológico la plataforma Google Classroom se basa en el entorno en el cual la sociedad se está adaptando a convivir ya con la virtualidad, se puede 
observar que aborda la era del conocimiento en la actualidad puesto que es una herramienta fundamental para la comunidad educativa, por tal motivo la investigación es de carácter relevante para la educación y se podrá realizar un análisis no experimental por medio de un enfoque cuantitativo, cohorte transversal siendo así un aporte para la ciencia y la tecnología.

Para Hernández et al. (2010) el enfoque cuantitativo ha sido más relevante en cuanto a las ciencias de tal forma que son llamadas como exactas o naturales; en cuanto a la presente investigación se utilizará un enfoque cuantitativo; las técnicas a utilizar serán las encuestas; el instrumento será el cuestionario con el modelo de Likert, se realizó un análisis cuantitavo por medio de una estadistica descriptiva analizado mediante el Alfa de Cronbach obteniendo un índice de 0,846 reflejando que es un aceptable índice de fiabilidad. Se realizó un análisis para determinar si son o no paramétricas, analizadas cada una de las preguntas indica que los resultados son paramétricos, realizado por medio de pruebas de normalidad (Kolmogorov-Smirnov). De acuerdo a la investigación realizada de alto impacto educativo a nivel de nuestra sociedad, se tomó en consideración a la escuela de Educación Básica 4 de noviembre de la ciudad de Azogues, provincia del Cañar, país Ecuador, siendo su población la escuela con un número de estudiantes de 256 , con un respectivo muestreo aleatorio estratificado que es la Básica Superior comprendido octavo, noveno y décimo año con 65 estudiantes.

\section{RESULTADOS}

Por lo que respecta a los resultados se consideró una tabla de frecuencias donde tambien nos viene dado porcentajes representando que, los estudiantes si utilizaría la plataforma Google Classroom con una aceptación positiva de 78,5\% muy de acuerdo y $18,5 \%$ algo de acuerdo respectivamente resultando un $97 \%$ de la población establecida, estando la misma satisfecha como se puede observar frecuencias y porcentajes válidos que han sido analizados en la siguiente tabla: 
Tabla 1.

Utilización de Google Classroom.

\begin{tabular}{lllll}
\hline Google Classroom & Frecuencia & Porcentaje & $\begin{array}{l}\text { Porcentaje } \\
\text { válido }\end{array}$ & $\begin{array}{l}\text { Porcentaje } \\
\text { acumulado }\end{array}$ \\
\hline Muy de acuerdo & 51 & 78,5 & 78,5 & 78,5 \\
Algo de acuerdo & 12 & 18,5 & 18,5 & 96,9 \\
Neutral & 1 & 1,5 & 1,5 & 98,5 \\
En desacuerdo & 1 & 1,5 & 1,5 & 100,0 \\
Total & 65 & 100,0 & 100,0 & \\
\hline
\end{tabular}

Fuente: Encuesta.

En referencia a la tabla 2 la importancia de utilizar en la actualidad la plataforma Google Classroom de manera positiva es de un $68,2 \%$ muy importante y $30,8 \%$ algo importante respectivamente resultando un 100\% siendo una aceptación en su totalidad de la población investigada, de esta forma es de gran impacto para quien está dirigida, lo cual indica cuantitativamente más detallada cada uno de sus porcentajes válidos y frecuencias.

\section{Tabla 2.}

Importancia de la plataforma.

\begin{tabular}{lllll}
\hline Google Classroom & Frecuencia & Porcentaje & $\begin{array}{l}\text { Porcentaje } \\
\text { válido }\end{array}$ & $\begin{array}{l}\text { Porcentaje } \\
\text { acumulado }\end{array}$ \\
\hline Muy importante & 45 & 68,2 & 68,2 & 69,2 \\
Importante & 20 & 30,8 & 30,8 & 100,0 \\
Total & 63 & 100,0 & 100,0 & \\
\hline
\end{tabular}

Fuente: Encuesta.

Tomando en consideración la tabla 3, analizado cada una de sus frecuencias y porcentajes menciona que si cumpliría las actividades pedagógicas por medio de una plataforma Google Classroom: obteniendo un $63,1 \%$ con muy de acuerdo y un $33,8 \%$ algo de acuerdo, siendo un resultado muy positivo de $96,9 \%$ de la población designada que si cumplirían sus tareas. 
Hecho el depósito de ley: pp201602FA4721

ISSN-L: 2542-3029; ISSN: 2610-802X

Universidad Nacional Experimental Francisco de Miranda (UNEFM). Santa Ana de Coro. Venezuela

Marcos Damián Vivar-Vicuña; Juan Carlos Erazo-Álvarez

Tabla 3.

Cumpliría actividades pedagógicas por medio de una plataforma.

\begin{tabular}{llccc}
\hline Google Classroom & Frecuencia & Porcentaje & $\begin{array}{l}\text { Porcentaje } \\
\text { válido }\end{array}$ & $\begin{array}{c}\text { Porcentaje } \\
\text { acumulado }\end{array}$ \\
\hline Muy de acuerdo & 41 & 63,1 & 63,1 & 63,1 \\
Algo de acuerdo & 22 & 33,8 & 33,8 & 96,9 \\
Neutral & 2 & 3,1 & 3,1 & 100,0 \\
Total & 65 & 100,0 & 100,0 & \\
\hline
\end{tabular}

Fuente: Encuesta.

La tabla 4 demuestra de que los estudiantes consideran que si se debería trabajar con esta estrategia de aprendizaje con una aceptación de: muy importante con el $72,3 \%$ y con importante con el $26,2 \%$ siendo un resultado positivo para la investigación siendo en su gran mayoría con el 98,5\% demostrando que es de gran importancia para la educación.

Tabla 4.

Google Classroom será importante para trabajar de manera asincrónica.

\begin{tabular}{llccc}
\hline Google Classroom & Frecuencia & Porcentaje & Porcentaje & $\begin{array}{c}\text { Porcentaje } \\
\text { acumulado }\end{array}$ \\
\hline Muy importante & 47 & 72,3 & 72,3 & 72,3 \\
Importante & 17 & 26,2 & 26,2 & 98,5 \\
Neutral & 1 & 1,5 & 1,5 & 100,0 \\
Total & 65 & 100,0 & 100,0 & \\
\hline
\end{tabular}

Fuente: Encuesta.

\section{PROPUESTA}

En el modelo explicativo del uso de la plataforma Google Classroom para el mejor aprovechamiento en el ámbito pedagógico por parte de los estudiantes, se efectuó con un enfoque en cuatro espacios establecidos: tablón, trabajo de clase, personas y calificación (Figura 1). 


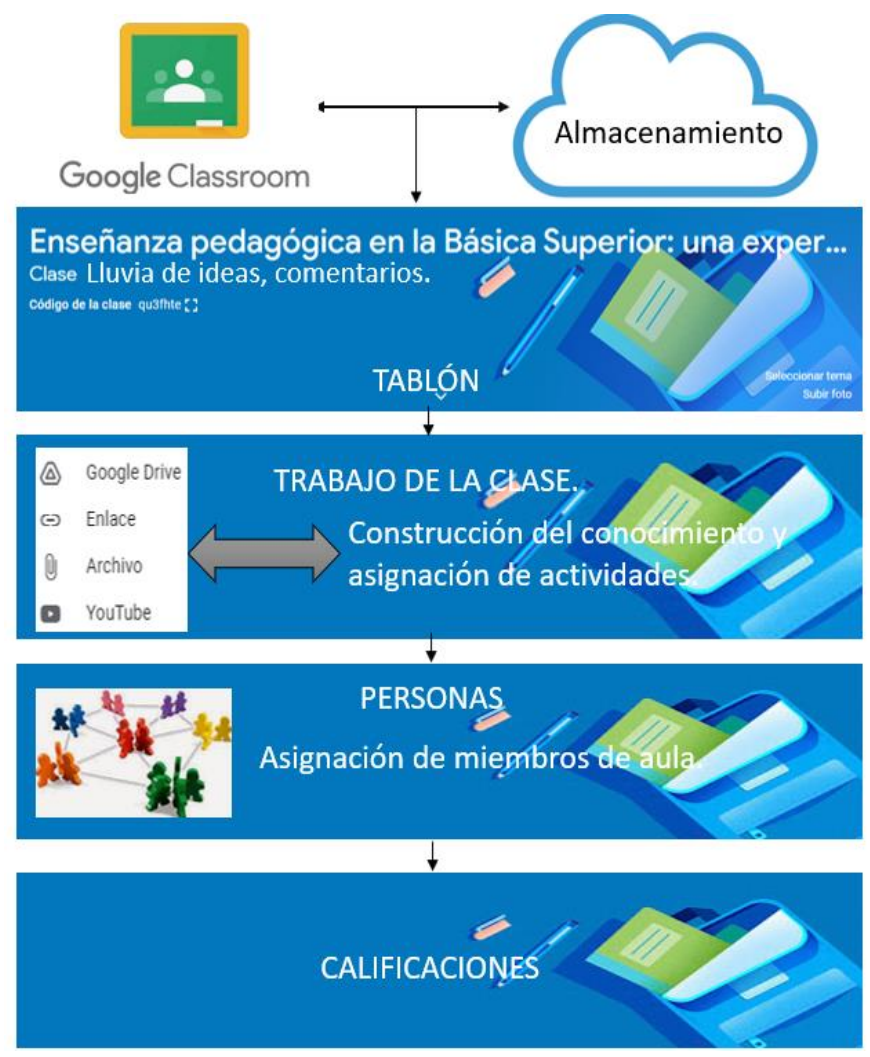

Figura 1. Modelo explicativo del uso de la plataforma Google Classroom Elaboración: Los autores.

A continuación se describe cada uno de sus elemenetos:

Tablón: es una de las herramientas mas importartes ya que es una presentación inicial de los que vamos a realizar en los siguientes elementos, por que puede servir como un recortadorio de la clase anterior, una comunicación de forma colavorativa por medio de comentarios, es decir interactuar estudiantes-profesor y profesorestudiantes, como tambien la realización de una lluvia de ideas sobre un tema propuesto por el docente.

Trabajo en clase: es la segunda fase para la utilización es el flujo de contenido donde encuentra ya le creación o llamado tambien construcción de conocimientos categorisando y guardando instrumentos de manera muy ordenada, mediante videos, enlaces, archivos y cualquier tipo de archivo adicional que se le pueda subir a la nube 


\section{CIENCIAMATRIA \\ Revista Interdisciplinaria de Humanidades, Educación, Ciencia y Tecnología \\ Año VII. Vol. VII. N¹3. Julio - Diciembre. 2021 \\ Hecho el depósito de ley: pp201602FA4721 \\ ISSN-L: 2542-3029; ISSN: 2610-802X \\ Universidad Nacional Experimental Francisco de Miranda (UNEFM). Santa Ana de Coro. Venezuela}

Marcos Damián Vivar-Vicuña; Juan Carlos Erazo-Álvarez

DRIVE, como material pedagógico para cada uno los estudiantes de acuerdo a las necesidades.

Personas: en el tercer apartado que refiere a miembros de la clase se puede asignar el número de estudiantes y docentes que desee, tomando en consideracion que una aula es de 30 a 40 estudiantes, de tal forma de poder trabajar ya sea de forma individual o colaborativa. Tambien se puede agregar a los estudiantes mediante un código llevandonos a evitar tiempo agregandoles por correos y puede acceder cualquier persona mediante el codigo asignado por Classroom.

Calificaciones: en cuanto a calificaciones se lleva a cabo una configuracion programada por el docente, la misma que va la fecha y hora limite para la presentación y la puntuación tanto en cada pregunta será asignada de acuerdo al grado de dificultad propuesto, como tambien el total de la nota se puede evaluar con varios instrumentos llegando a su vez a la etapa final y cumplimiento de la clas por mediio de la plataforma.

\section{CONCLUSIONES}

La investigación se realizó puesto que los conocimientos obtenidos por el individuo cada vez son mas amplios, los estudiantes-docentes y docentes-estudiantes intercambian ideas, procesos de aprendizaje, es decir trasferencia de saberes mediante herramientas digitales como medios de comunicación en la comunidad educativa de esta manera Google Classroom es una herramienta digital creada para el beneficio exclusivamente hoy en día de la educación.

Se pudo investigar por medio de un análisis cuantitativo basado en el entorno de la sociedad educativa puesto que se está conviviendo con la virtualidad se realizó un análisis no experimental de cohorte transversal por medio de un cuestionario a la población designada de estudiantes con un muestreo aleatoria estratificado en el cual los investigados están entusiasmados en conocer Google Classroom para su actividad pedagógica. 


\section{CIENCIAMATRIA}

Revista Interdisciplinaria de Humanidades, Educación, Ciencia y Tecnología

Año VII. Vol. VII. N¹3. Julio - Diciembre. 2021

Hecho el depósito de ley: pp201602FA4721

ISSN-L: 2542-3029; ISSN: 2610-802X

Universidad Nacional Experimental Francisco de Miranda (UNEFM). Santa Ana de Coro. Venezuela

Marcos Damián Vivar-Vicuña; Juan Carlos Erazo-Álvarez

Por medio de un modelo explicativo del uso de la plataforma Google Classroom los estudiantes de la población asignada pueden mejorar en la educación en el ámbito pedagógico ya sea de manera virtual o presencial con las aportaciones de conocimiento individuales o de manera colaborativa siendo un intercambio de cualidades y virtudes de cada uno de los estududiantes de la comunidad educativa, contribuyendo un mejor futuro para una calidad educativa.

En lo referente a la utilización de la plataforma hacia la población asignada para esta investigación, se obtuvo que se encuentran muy satisfechos de acuerdo a los resultados analizados los estudiantes están en la capacidad y con las ganas de aprender cosas nuevas que se les presenta en el ámbito educativo ya sea de para trabajar de manera sincrónica o asincrónica respectivamente como es la plataforma pedagógica Google Classroom.

Por último, se ha determinada que en la actualidad la plataforma Google Classroom es de vital importancia para desarrollarse en el ámbito educativo puesto que se está enfrentando a múltiples modalidades de estudio presentes hoy en esta nueva era del conocimiento y la tecnología, destacando que cada día se tiene el deseo de seguir superándose en cuanto a conocimientos y también desarrollándose el individuo a nivel profesional.

\section{REFERENCIAS}

Aznar, I., Romero, J., \& Rodríguez, A. (2018). La tecnología móvil de Realidad Virtual en educación: una revisión del estado de la literatura científica en España Virtual [The mobile technology of Virtual Reality in education: a review of the state of the scientific literature in Virtual Spain]. Edmetic, 7(1), 256-274.

Cacheiro, M. (2011). Recursos educativos tic de información, colaboración y aprendizaje. [Educational ICT information, collaboration and learning resources]. International Journal of Circumpolar Health, 39, 69-81. https://doi.org/10.3402/ijch.v71i0.19265

Cano, L., \& Olda, M. (2012). Antecedentes internacionales y nacionales de las TIC a nivel superior: su trayectoria en Panamá [International and national background of ICT at a higher level: his trajectory in Panama]. Actualidades Investigativas En Educación, 12(3), 1-25. https://doi.org/10.15517/aie.v12i3.10303 


\author{
CIENCIAMATRIA \\ Revista Interdisciplinaria de Humanidades, Educación, Ciencia y Tecnología \\ Año VII. Vol. VII. NN13. Julio - Diciembre. 2021 \\ Hecho el depósito de ley: pp201602FA4721 \\ ISSN-L: 2542-3029; ISSN: 2610-802X \\ Universidad Nacional Experimental Francisco de Miranda (UNEFM). Santa Ana de Coro. Venezuela
}

Marcos Damián Vivar-Vicuña; Juan Carlos Erazo-Álvarez

Gomez, G., \& Valdivia, M. (2020). Aplicación de la plataforma Google Classroom en los estudiantes de tercer año de secundaria. [Application of the Google Classroom platform in third year high school students]. 2507(February), 1-53. https://n9.cl/2bxjv

Hernández, M. (2017). Impacto de las TIC en la educación: Retos y Perspectivas. [Impact of ICT on Education: Challenges and Perspectives]. Pixel-Bit, Revista de Medios y Educacion, 5(52), 81-96. https://doi.org/10.12795/pixelbit.2018.i52.06

Hernández, R., Fernández, C., \& Baptista, P. (2010). Metodología de la investigacion. [Investigation methodology]. https://n9.cl/zg165

LOEI. (2013). Ley Orgánica De Educación Intercultural Titulo I De Los Principios Generales Capítulo Único Del Ámbito, Principios Y Fines. https://n9.cl/k1323

Monge, J. (2014). Estrategias participativas para el desarrollo del razonamiento lógico, en el aprendizaje de matemática de los alumnos de quinto, sexto, séptimo y octavo años de educación básica [Participatory strategies for the development of logical reasoning, in the le. Universidad Técnica De Cotopaxi Facultad, 1, 101. https://n9.cl/as4wh

Navarrete, G., \& Mendieta, R. (2018). Las Tic Y La Educación Ecuatoriana En Tiempos De Internet: Breve Análisis. [TICs and Ecuadorian Education in the Times of the Internet: Brief Analysis]. Espirales, 2(15), 123-136. https://n9.cl/1xgzi

Páez-Barón, E., Corredor-Camargo, E., \& Fonseca-Carreño, J. (2016). Evaluación del uso de herramientas sincrónicas y asincrónicas en procesos de formación de las ciencias agropecuarias. [Evaluation of the use of synchronous and asynchronous tools in agricultural science training processes]. CIENCIA Y AGRICULTURA, 13(1), 77. https://doi.org/10.19053/01228420.4808

Paladines, C. (2016). Perspectivas de cambio en la Educación Básica y en el Bachillerato. Ecuador: 2007-2013. [Prospects for change in the basic education and high school. Ecuador: 2007-2013]. La Educación Secundaria En Chile. Situación Actual y Proyecciones, 19(3), 13-31. https://n9.cl/nglc3

Peña, F., \& Otálora, N. (2018). Educación y tecnología: problemas y relaciones [Education and technology: problems and relationships]. Pedagogía y Saberes, 48, 59-70. https://doi.org/10.17227/pys.num48-7373

Poma Ramos, C. (2019). El uso del Virtual Classroom y la percepción del grado de utilidad en el aprendizaje por competencias de los estudiantes de la Universidad Nacional de Huancavelica Sede Lircay-2018 [The use of the Virtual Classroom and the perception of the degree of util. $1-133$. https://n9.cl/m78bd 
Puga Peña, L., \& Jaramillo Naranjo, L. (2015). Metodología activa en la construcción del conocimiento matemático [Active methodology in the construction of mathematical knowledge]. Sophía, 1(19), 291-314. https://doi.org/10.17163/soph.n19.2015.14

Rodríguez, M., Chaparro, S., \& Rojas, W. (2013). Scientific production of the journal Investigación y Educación en Enfermería during its 30 editing years.[Producción científica de la revista Investigación y Educación en Enfermería durante sus 30 años de edición]. Investigación y Educación En Enfermería, 31(3), 341-353. https://n9.cl/fchm0

Tabarez, N. (2010). Elaboración del conocimiento y patrones de sensibilidad estética en estudiantes de Arte, a través de su relación con espacios de realidad virtual.[Construction of knowledge and sensitivity aesthetics patterns in art students, through their relationship wi. Revista de Investigación, 34(69), 243-264. https://n9.cl/asv2d 